\title{
Charming top decays with a flavor changing neutral Higgs boson and $W W$ at hadron colliders
}

\author{
Rishabh Jain* and Chung Kao ${ }^{\dagger}$ \\ Homer L. Dodge Department of Physics and Astronomy, University of Oklahoma, \\ Norman, Oklahoma 73019, USA
}

(Received 4 January 2019; published 27 March 2019)

\begin{abstract}
We investigate the prospects for discovering a top quark decaying into one light Higgs boson $\left(h^{0}\right)$ along with a charm quark $(c)$ in top-quark pair production at the CERN LHC and future hadron colliders. A general two Higgs doublet model is adopted to study the signature of flavor changing neutral Higgs interactions with $t \rightarrow c h^{0}$, followed by $h^{0} \rightarrow W W^{*} \rightarrow \ell^{+} \ell^{-}+E_{T}$, where $h^{0}$ is the $C P$-even Higgs boson and $E_{T}$ stands for missing transverse energy from neutrinos. We study the discovery potential for this flavor changing neutral Higgs signal and physics background from dominant processes with realistic acceptance cuts as well as tagging and mistagging efficiencies. Promising results are found for the LHC running at 13 and $14 \mathrm{TeV}$ center-of-mass energy as well as future pp colliders at 27 and $100 \mathrm{TeV}$.
\end{abstract}

DOI: 10.1103/PhysRevD.99.055036

\section{INTRODUCTION}

The Standard Model has been very successful in explaining almost all experimental data to date, culminating in the discovery of the long-awaited standard Higgs boson at the CERN LHC [1,2]. The most important experimental goals of the LHC, future high-energy hadron colliders, and the International Linear Collider are to study the Higgs properties and to search for new physics beyond the Standard Model (SM) including additional Higgs bosons and flavor changing neutral Higgs (FCNH) interactions.

In the Standard Model, there is one Higgs doublet, which generates masses for both vector bosons and fermions. There is no explanation for the large differences among Yukawa couplings of fermions with the Higgs boson. In addition, there are no flavor changing neutral currents (FCNCs) mediated by gauge interactions or by Higgs interactions at the tree level. The top quark is the most massive elementary particle ever discovered. The fact that the Higgs boson $\left(h^{0}\right)$ is lighter than the top quark $\left(m_{t}>M_{h}\right)$ makes it possible for the top quark to decay into the Higgs boson along with a charm quark $\left(t \rightarrow c h^{0}\right)$ kinematically. At the one-loop level, the branching fraction of $t \rightarrow c h^{0}$ is $3 \times 10^{-15}$ for $M_{h}=125 \mathrm{GeV}$ [3-5]. If this decay mode is detected in the near future, it would indicate

\footnotetext{
*Rishabh.Jain@ou.edu

†Chung.Kao@ou.edu

Published by the American Physical Society under the terms of the Creative Commons Attribution 4.0 International license. Further distribution of this work must maintain attribution to the author(s) and the published article's title, journal citation, and DOI. Funded by SCOAP ${ }^{3}$.
}

a large tree-level coupling or a significant enhancement from beyond SM loop effects.

A general two Higgs doublet model (2HDM) usually contains FCNH interactions if there is no discrete symmetry to turn off tree-level FCNCs [6,7]. In 1991, it was pointed out that top-charm FCNH coupling could be prominent [8] if the Yukawa couplings of fermions and the Higgs boson are comparable to the geometric mean of their mass [9]. A special two Higgs doublet model for the top quark (T2HDM) [10] might provide a reasonable explanation as to why the top quark is much more massive than other elementary fermions. In the T2HDM, the top quark is the only elementary fermion acquiring its mass from a special Higgs doublet $\left(\phi_{2}\right)$ with a large vacuum expectation value $\left(v_{2} \gg v_{1}\right)$. Since the up and charm quarks couple to another Higgs doublet $\left(\phi_{1}\right)$, there are FCNH interactions among the up-type quarks. The downtype quarks have the same interactions as those in the SM.

In a general two Higgs doublet model, there are five physical Higgs bosons: two $C P$-even scalars $h^{0}$ (lighter) and $H^{0}$ (heavier), a $C P$-odd pseudoscalar $\left(A^{0}\right)$, and a pair of singly charged Higgs boson $\left(H^{ \pm}\right)$. To study FCNH interactions in a general 2HDM, we employ the Lagrangian with Higgs bosons and fermions [11,12],

$$
\begin{aligned}
\mathcal{L}_{Y}= & \frac{-1}{\sqrt{2}} \sum_{F=U, D, L} \bar{F}\left\{\left[\kappa^{F} s_{\beta-\alpha}+\rho^{F} c_{\beta-\alpha}\right] h^{0}\right. \\
& \left.+\left[\kappa^{F} c_{\beta-\alpha}-\rho^{F} s_{\beta-\alpha}\right] H^{0}-i \operatorname{sgn}\left(Q_{F}\right) \rho^{F} A^{0}\right\} P_{R} F \\
& -\bar{U}\left[V \rho^{D} P_{R}-\rho^{U_{\dagger}^{\dagger}} V P_{L}\right] D H^{+}-\bar{\nu}\left[\rho^{L} P_{R}\right] L H^{+}+\text {H.c. }
\end{aligned}
$$


where $\quad P_{L, R} \equiv\left(1 \mp \gamma_{5}\right) / 2, \quad c_{\beta-\alpha}=\cos (\beta-\alpha), \quad s_{\beta-\alpha}=$ $\sin (\beta-\alpha), \alpha$ is the mixing angle between neutral Higgs scalars, $\tan \beta \equiv v_{2} / v_{1}$ [7], $Q_{F}$ is the charge of a fermion, and $\kappa$ matrices are diagonal and fixed by fermion masses to $\kappa^{F}=\sqrt{2} m_{F} / v$ with $v \simeq 246 \mathrm{GeV}$, while $\rho$ matrices have both diagonal and off-diagonal elements with free parameters.

The LHC has become a top-quark factory. The production cross section of the top-quark pair $\left(\sigma_{t t}\right)$ is approximately $820 \mathrm{pb}$ in pp collisions with a $13 \mathrm{TeV}$ c.m. energy $(\sqrt{s})$, and it becomes $\sigma_{t t} \simeq 970 \mathrm{pb}$ at $\sqrt{s}=14 \mathrm{TeV}$ [13-17]. For an integrated luminosity of $\mathcal{L}=100 \mathrm{fb}^{-1}$ at $\sqrt{s}=13 \mathrm{TeV}$, the LHC has produced more than $8 \times 10^{7}$ top-quark pairs $(t \bar{t})$ for $m_{t} \simeq 173.2 \mathrm{GeV}[18,19]$. For the same integrated luminosity at $\sqrt{s}=14 \mathrm{TeV}$, the number of $(t \bar{t})$ pairs generated would increase to about $1 \times 10^{8}$. Thus, the LHC will provide great opportunities to study electroweak symmetry breaking as well as other important properties of the top quark and the Higgs boson.

Most ATLAS and CMS measurements of the $125 \mathrm{GeV}$ Higgs boson $\left(h^{0}\right)$ are consistent with expectations for the Standard Model. The branching fractions of the standard Higgs boson are presented in Table I [20-22]. In a general two Higgs doublet model, let us consider the light Higgs scalar $\left(h^{0}\right)$ as the SM Higgs boson in the alignment limit $[23,24]$.

It is clear that the most probable decay channels are $b \bar{b}$ and $W W$ with branching fractions $\mathcal{B}\left(h^{0} \rightarrow b \bar{b}\right) \simeq 0.58$ and $\mathcal{B}\left(h^{0} \rightarrow W W^{*}\right) \simeq 0.22$ as shown in Table I. However, the light Higgs boson was first discovered with $h^{0} \rightarrow \gamma \gamma$ and $h^{0} \rightarrow Z Z^{*} \rightarrow 4 \ell$ because these channels have less background and better mass resolutions. In the past few years, several theoretical studies and experimental searches have been completed for the charming top FCNH decay $t \rightarrow c h^{0}$ with (a) $h^{0} \rightarrow b \bar{b}$ [25-28], (b) $h^{0} \rightarrow Z Z^{*}$ [29], (c) $h^{0} \rightarrow \gamma \gamma$ [28,30], and (d) Higgs decays into multileptons [31-33]. Recently, the ATLAS Collaboration has placed tight limits on the FCNH branching fraction for $t \rightarrow c h^{0}$ and the Yukawa coupling $\lambda_{\text {tch }}$ with the Higgs boson decaying into multileptons [34]

TABLE I. Branching fractions and partial decay widths of the light $C P$-even Higgs boson $\left(h^{0}\right)$ of a general two Higgs doublet model in the alignment limit $\left(h^{0} \simeq h_{\mathrm{SM}}^{0}\right)$. For simplicity, let us take $\rho_{f f} \simeq \kappa_{f}=\sqrt{2} m_{f} / v$. Widths are in mega-electron-volt units, with $\Gamma_{h^{0}}^{\mathrm{SM}} \simeq 4.1 \mathrm{MeV}$ [20].

\begin{tabular}{lclc}
\hline \hline Decay channel & $\mathcal{B}^{\mathrm{SM}}$ & $\Gamma(\mathrm{MeV})$ & Comment \\
\hline$b b$ & $57.5 \%$ & 2.35 & $\rho_{b b} \simeq \kappa_{b}$ \\
$W W^{*}$ & $21.6 \%$ & 0.89 & $\sin (\beta-\alpha) \simeq 1$ \\
$g g$ & $8.56 \%$ & 0.35 & $\rho_{t t} \simeq \kappa_{t} \sim 1$ \\
$\tau \tau$ & $6.30 \%$ & 0.26 & $\rho_{\tau \tau} \simeq \kappa_{\tau}$ \\
$Z Z^{*}$ & $2.67 \%$ & 0.11 & $\sin (\beta-\alpha) \simeq 1$ \\
$\gamma \gamma$ & $0.23 \%$ & 0.094 & $W$ loop and fermion loops \\
\hline \hline
\end{tabular}

$$
\mathcal{B}\left(t \rightarrow c h^{0}\right) \leq 0.16 \%, \quad \text { and } \quad \lambda_{t c h} \leq 0.077,
$$

for the effective Lagrangian

$$
\mathcal{L}_{\text {eff }}=-\frac{\lambda_{t c h}}{\sqrt{2}} \bar{c} t h^{0}+\text { H.c.. }
$$

The LHC limits for the branching ratios can be translated to a limit on the flavor changing Yukawa coupling by a simple rescaling. It is a good approximation to consider a simple numerical relation between the FCNH Yukawa coupling $\left(\lambda_{t c h}\right)$ and the branching fraction of $t \rightarrow c h^{0}$ [35],

$$
\lambda_{\text {tch }} \simeq 1.92 \times \sqrt{\mathcal{B}\left(t \rightarrow c h^{0}\right)} .
$$

In this article, we focus on the discovery potential of the LHC in the search for the FCNH top decay $t \rightarrow c h^{0}$ followed by $h^{0} \rightarrow W W^{*} \rightarrow \ell^{+} \ell^{-} \nu \bar{\nu}$. We have evaluated production rates with full tree-level matrix elements including Breit-Wigner resonances for both the signal and the physics background. In addition, we optimize the acceptance cuts to effectively reduce the background with realistic $b$-tagging and mistagging efficiencies. Promising results are presented for the LHC with $\sqrt{s}=13 \mathrm{TeV}$ and $\sqrt{s}=14 \mathrm{TeV}$ as well as for future hadron colliders at $\sqrt{s}=27 \mathrm{TeV}$ and $100 \mathrm{TeV}$, for high luminosities (HLs) [36-39] of $L=300$ and $3000 \mathrm{fb}^{-1}$. Section II shows the production cross sections for the Higgs signal and the dominant background as well as our strategy to determine the reconstructed masses for the top quark and the Higgs boson. Realistic acceptance cuts are discussed in Sec. III. Section IV presents the discovery potential at the LHC for $\sqrt{s}=13$ and $14 \mathrm{TeV}$ as well as for future hadron colliders with $\sqrt{s}=27$ and $100 \mathrm{TeV}$. Our optimistic conclusions are drawn in Sec. V.

\section{HIGGS SIGNAL AND PHYSICS BACKGROUND}

In this section, we present the cross section for the FCNH Higgs signal in pp collisions $\left(p p \rightarrow t \bar{t} \rightarrow t c h^{0} \rightarrow\right.$ bjjcle $\ell \bar{\nu}+X, \ell=e, \mu)$ as well as for the dominant physics background processes. Figure 1 shows the Feynman diagram of top-quark pair production in $\mathrm{pp}$ collisions from gluon fusion and quark-antiquark fusion, followed by one top quark decaying into a Higgs boson and a charm quark, while the other top quark decays into $b W \rightarrow b j j$.

\section{A. Higgs signal in top decay}

Applying the Lagrangian in Eq. (1) with general Yukawa interactions for the light Higgs boson and fermions, we obtain the decay width of $t \rightarrow c h^{0}$, 


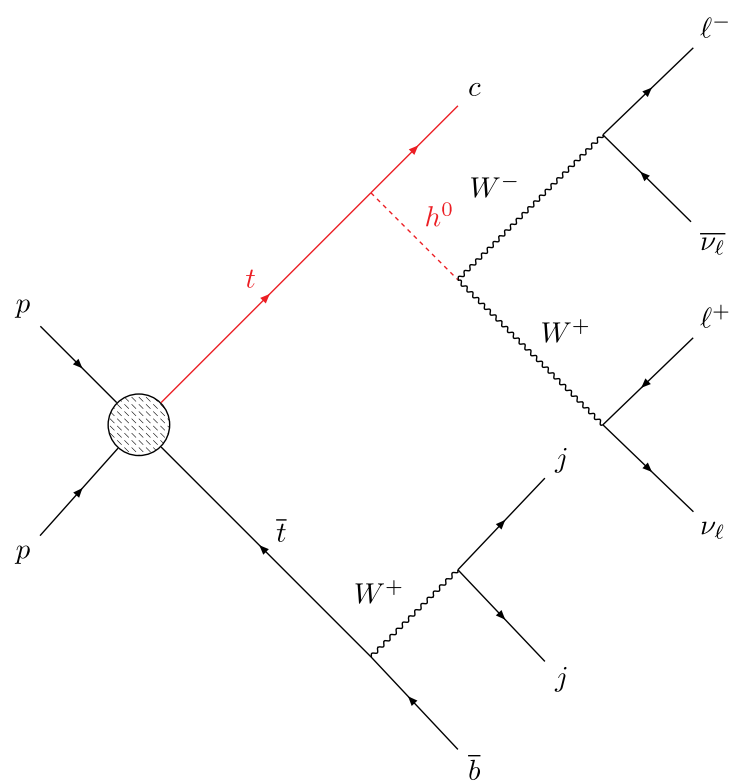

FIG. 1. Feynman diagram for $p p \rightarrow t \bar{t} \rightarrow b j j c h^{0}+X \rightarrow$ bjjc $\ell^{+} \ell^{-} \nu \bar{\nu} X$, where $\ell=e$ or $\mu$.

$$
\begin{aligned}
\Gamma_{t \rightarrow c h^{0}}= & \frac{c_{\beta-\alpha}^{2} m_{t}}{32 \pi}\left[\left(1+r_{c}^{2}-r_{h}^{2}\right) \frac{\left(\left|\rho_{c t}\right|^{2}+\left|\rho_{t c}\right|^{2}\right)}{2}\right. \\
& +r_{c}\left(\rho_{t c}^{*} \rho_{c t}^{*}+\rho_{t c} \rho_{c t)}\right] \lambda^{1 / 2}\left(1, r_{c}^{2}, r_{h}^{2}\right),
\end{aligned}
$$

where $c_{\beta-\alpha}=\cos (\beta-\alpha), r_{h}=M_{h} / m_{t}, r_{c}=m_{c} / m_{t}$, and

$$
\lambda(x, y, z)=x^{2}+y^{2}+z^{2}-2 x y-2 x z-2 y z .
$$

Let us define two variables,

$\tilde{\rho}_{t c}=\sqrt{\frac{\left|\rho_{t c}\right|^{2}+\left|\rho_{c t}\right|^{2}}{2}}, \quad$ and $\quad \epsilon_{c}=\rho_{t c}^{*} \rho_{c t}^{*}+\rho_{t c} \rho_{c t}$.

Combining LHC Higgs data and $B$ physics, a recent study found constraints $\rho_{t c} \leq 1.5$ and $\rho_{c t} \leq 0.1$ [40]. That implies $\epsilon_{c} \lesssim 0.2 \tilde{\rho}_{t c}$ for $\rho_{t c} \simeq 1$. Hence, we can write our decay width as

$\Gamma_{t \rightarrow c h^{0}}=\frac{c_{\beta-\alpha}^{2} m_{t}}{32 \pi}\left[\left(1+r_{c}^{2}-r_{h}^{2}\right)\left|\tilde{\rho}_{t c}\right|^{2}+\epsilon_{c} r_{c}\right] \times \lambda^{1 / 2}\left(1, r_{c}^{2}, r_{h}^{2}\right)$.

Using $m_{t}=173.2 \mathrm{GeV}, \quad M_{h}=125.1 \mathrm{GeV}$, and $m_{c}=$ $1.42 \mathrm{GeV}$ [20], we obtain

$\left.\Gamma_{t \rightarrow c h^{0}}=\frac{c_{\beta-\alpha}^{2} m_{t}}{32 \pi}\left[0.48\left|\tilde{\rho}_{t c}\right|^{2}+0.008 \epsilon_{c}\right)\right] \times \lambda^{1 / 2}\left(1, r_{c}^{2}, r_{h}^{2}\right)$.

Since we have $m_{c} \ll m_{t}, r_{c} \ll 1$, and $\epsilon_{c} \lesssim 0.2\left|\tilde{\rho}_{t c}\right|$, it is a very good approximation to consider

$$
\Gamma_{t \rightarrow c h^{0}} \simeq \frac{c_{\beta-\alpha}^{2} m_{t}}{32 \pi}\left[\left(1-r_{h}^{2}\right)\left|\tilde{\rho}_{t c}\right|^{2}\right] \times \lambda^{1 / 2}\left(1, r_{c}^{2}, r_{h}^{2}\right) .
$$

For typical values of parameters $\cos (\beta-\alpha)=0.1,\left|\rho_{t c}\right| \sim 1$ and $\left|\rho_{c t}\right| \sim 0.1$, we have

$$
\Gamma_{t \rightarrow c h^{0}} \simeq 0.394\left(c_{\beta-\alpha}^{2}\left|\tilde{\rho}_{t c}\right|^{2}\right) \simeq 0.00197 \mathrm{GeV},
$$

and

$$
\mathcal{B}\left(t \rightarrow c h^{0}\right) \simeq 0.00132 .
$$

For simplicity, we may adopt the effective Lagrangian to study FCNH Yukawa interactions for the light $C P$-even Higgs boson $\left(h^{0}\right)$ with the top quark $(t)$ and the charm quark $(c)$,

$$
\mathcal{L}=-g_{h t c} \bar{c} t h^{0}+\text { H.c. }
$$

where

$$
g_{h t c}=\frac{1}{\sqrt{2}} \tilde{\rho}_{t c} \cos (\beta-\alpha)=\frac{1}{\sqrt{2}} \lambda_{t c h} .
$$

It is the effective coupling of the FCNH Yukawa coupling.

Then, the decay width for $t \rightarrow c h^{0}$ [8] becomes

$$
\begin{aligned}
\Gamma\left(t \rightarrow c \phi^{0}\right)= & \frac{\left|g_{h t c}\right|^{2}}{16 \pi} \times\left(m_{t}\right) \times\left[1+r_{c}^{2}-r_{h}^{2}\right] \\
& \times \sqrt{1-\left(r_{h}+r_{c}\right)^{2}} \sqrt{1-\left(r_{h}-r_{c}\right)^{2}} .
\end{aligned}
$$

We assume that the total decay width of the top quark is

$$
\Gamma_{t}=\Gamma(t \rightarrow b W)+\Gamma\left(t \rightarrow c h^{0}\right) .
$$

Then, the branching fraction of $t \rightarrow c h^{0}$ becomes

$$
\mathcal{B}\left(t \rightarrow c h^{0}\right)=\frac{\Gamma\left(t \rightarrow c h^{0}\right)}{\Gamma_{t}}
$$

For a case study, let us take the FCNH Yukawa couplings to be the geometric mean of the Yukawa couplings of the quarks that is also known as the Cheng-Sher (CS) ansatz [9],

or

$$
\lambda_{t c h}(\mathrm{CS})=\sqrt{2} g_{h t c}(\mathrm{CS})=\frac{\sqrt{2 m_{t} m_{c}}}{v} \simeq 0.0901,
$$

with $m_{t}=173.2 \mathrm{GeV}$ and $m_{c}=1.42 \mathrm{GeV}$. Then, the branching fraction of $t \rightarrow c h^{0}$ becomes $\mathcal{B}\left(t \rightarrow c h^{0}\right)=$ $2.2 \times 10^{-3}$ for $M_{h}=125.1 \mathrm{GeV}$. In general, we will 
consider $g_{h t c}=\tilde{\rho}_{t c} \cos (\beta-\alpha) / \sqrt{2}$ with $\tilde{\rho}_{t c}$ and $\cos (\beta-\alpha)$ as free parameters.

We employ the programs MadGraph [41,42] and HELAS [43] to evaluate the exact matrix element for the FCNH signal in top decays from gluon fusion and quark-antiquark annihilation,

$$
\begin{aligned}
& g g, q \bar{q} \rightarrow t \bar{t} \rightarrow t \bar{c} h^{0} \rightarrow b j j \bar{c} \ell^{+} \ell^{-} \nu \bar{\nu}, \quad \text { and } \\
& g g, q \bar{q} \rightarrow \bar{t} t \rightarrow \bar{t} c h^{0} \rightarrow \bar{b} j j c \ell^{+} \ell^{-} \nu \bar{\nu},
\end{aligned}
$$

where $\ell=e$ or $\mu$. The cross section of the Higgs signal in FCNH top decays at the LHC and future hadron colliders for $p p \rightarrow t \bar{t} \rightarrow t c h^{0} \rightarrow b j j c \ell^{+} \ell^{-} \nu \bar{\nu}+X$ is evaluated with the parton distribution functions of CT14LO $[44,45]$ with a common value $Q=M_{t \bar{t}}$ equal to the invariant mass of $t \bar{t}$ for the renormalization scale $\left(\mu_{R}\right)$ and the factorization scale $\left(\mu_{F}\right)$. This choice of scale leads to a $\mathrm{K}$ factor of approximately 1.8 for top-quark pair production. We have used the computer program Top++ [17] to evaluate higher-order corrections. In addition, we have checked the tree-level signal cross section with narrow width approximation. That is, the cross section $\sigma\left(p p \rightarrow t \bar{t} \rightarrow t c h^{0} \rightarrow b j j c \ell^{+} \ell^{-} \nu \bar{\nu}+X\right)$ is calculated as the product of cross section times branching fractions:

$$
\begin{aligned}
& \sigma(p p \rightarrow t \bar{t} \rightarrow b j j \bar{t}+X) \times \mathcal{B}\left(t \rightarrow c h^{0}\right) \times \mathcal{B}\left(h^{0} \rightarrow W^{+} W^{-}\right) \\
& \quad \times\left[\mathcal{B}\left(W \rightarrow \ell \nu_{\ell}\right)\right]^{2} .
\end{aligned}
$$

In our analysis, we consider the FCNH signal from both $t \bar{t} \rightarrow t \bar{c} h^{0} \rightarrow b j j \bar{c} \ell^{+} \ell^{-} \nu_{\ell} \overline{\nu_{\ell}}$ and $\bar{t} \rightarrow c h^{0} \bar{t} \rightarrow \bar{b} j j c \ell^{+} \ell^{-} \nu_{\ell} \overline{\nu_{\ell}}$. In every event, we require that there should be one $b$ jet and three light jets ( $j=u, d, s, c$, or $g$ in physics background). In addition, there are two leptons $(\ell=e$ or $\mu)$ and neutrinos, which will be lead to missing transverse energy $\left(\mathscr{E}_{T}\right)$. Unless explicitly specified, $q$ generally denotes a quark $(q)$ or an antiquark $(\bar{q})$, and $\ell$ will represent a lepton $\left(\ell^{-}\right)$or antilepton $\left(\ell^{+}\right)$. That means our FCNH signal leads to the final state of $b j j c \ell^{+} \ell^{-} \nu_{\ell} \overline{\nu_{\ell}}$ or $b j j j \ell^{+} \ell^{-}+E_{T}$.

\section{B. Physics background}

The dominant physics background to the final state of bjjc $\ell^{+} \ell^{-} \nu \bar{\nu}$ comes from top-quark pair production along with two light jets $(t \bar{t} j j), \quad p p \rightarrow t \bar{t} j j \rightarrow b \bar{b} j j W W \rightarrow$ $b \bar{b} j j \ell^{+} \ell^{-} \nu \bar{\nu}+X$, where every top quark decays into a $b$ quark as well as a $W$ boson $(W \rightarrow \ell \nu)$ and a $b$ jet is misidentified as a $c$ jet. We have also considered backgrounds from $p p \rightarrow t \bar{t} W \rightarrow b \bar{b} j j W W \rightarrow b \bar{b} j j \ell^{+} \ell^{-} \nu \bar{\nu}+X$ with one $W$ boson decaying into $j j$, and $p p \rightarrow b \bar{b} j j W W \rightarrow$ $b \bar{b} j j \ell^{+} \ell^{-} \nu \bar{\nu}+X$, excluding the contribution from $t \bar{t} j j$ and $\bar{t} \bar{t} W$. In addition, we have included $p p \rightarrow c \bar{c} j j W W \rightarrow$ $c \bar{c} j j \ell^{+} \ell^{-} \nu \bar{\nu}+X$ and $p p \rightarrow j j j j W W \rightarrow j j j j \ell^{+} \ell^{-} \nu \bar{\nu}+X$, where $j=u, d, s$, or $g$. We evaluate the cross section of physics background in pp collisions with proper tagging and mistagging efficiencies. In our analysis, we adopt updated ATLAS tagging efficiencies [46,47]: the $b$-tagging efficiency is $\sim 70 \%$, the probability that a $c$ jet is mistagged as a $b$ jet $\left(\epsilon_{c}\right)$ is approximately $14 \%$, and the probability that any other jet is mistagged as a $b$ jet $\left(\epsilon_{j}\right)$ is $1 \%$.

\section{Mass reconstruction}

In this subsection, we demonstrate that the proposed Higgs signal comes from top-quark pair production with $t \bar{t} \rightarrow$ bjjch $h^{0} \rightarrow$ bjjc $\ell^{+} \ell^{-}+E_{T}$. We discuss our strategy to determine the reconstructed top mass as the invariant mass of $b j j$ from $t \rightarrow b W \rightarrow b j j$ along with another top quark decaying into a Higgs boson and a charm quark $t \rightarrow c h^{0}$. Furthermore, we employ cluster transverse mass distributions for $\ell^{+} \ell^{-}$and $c \ell^{+} \ell^{-}$with missing transverse energy $\left(E_{T}\right)$ from neutrinos. These distributions have broad peaks near $M_{T}\left(\ell \ell, E_{T}\right) \sim M_{h}$ and $M_{T}\left(c \ell \ell, E_{T}\right) \sim m_{t}$ respectively as the kinematic characteristics of $t \rightarrow c h^{0} \rightarrow c \ell^{+} \ell^{-}+E_{T}$. Applying suitable cuts on the cluster transverse mass $M_{T}\left(\ell \ell, E_{T}\right)$ as well as $M_{T}\left(c \ell \ell, E_{T}\right)$, we can greatly reduce the physics background and enhance the statistical significance for the Higgs signal.

In our analysis, we assume that the FCNH signal comes from top-quark pair production with one top quark decaying into a charm quark and a Higgs boson $\left(t \rightarrow c h^{0} \rightarrow c W W \rightarrow c \ell^{+} \nu \ell^{-} \bar{\nu}\right)$ while the other decays hadronically $(t \rightarrow b W \rightarrow b j j)$. In every event, there is one tagged $b$ jet and three light jets. Let us choose the pair of light jets that minimize $\left|M_{j j}-m_{W}\right|$ and $\left|M_{b j j}-m_{t}\right|$ as $j_{1} j_{2}$ and label the other jet as $j_{3} \simeq c$. That means, for a correctly reconstructed event, $j_{1}$ and $j_{2}$ are the products of a $W$ decay such that their invariant mass distribution peaks at $M_{j_{1} j_{2}} \simeq m_{W}$. For a background event, one $b$ is likely coming from the top decay $t \rightarrow b W \rightarrow b j j$, while the other is either a mistagged $c$ or a light quark jet coming from $W$ decay or a real $b$ quark coming from the decay of $\bar{t}$.

We present the invariant mass distributions for $M_{j_{1} j_{2}}$ and $M_{b j_{1} j_{2}}$ in Fig. 2 for the Higgs signal $\left(\bar{t} \rightarrow t c h^{0}\right)$ and the dominant background $(t \bar{t} j j)$ with basic cuts from CMS [48],

(a) $p_{T}(b, j)>25 \mathrm{GeV}$,

(b) $p_{T}\left(\ell_{1}\right)>25 \mathrm{GeV}, \quad p_{T}\left(\ell_{2}\right)>15 \mathrm{GeV}$,

(c) $E_{T}>25 \mathrm{GeV}$,

(d) $|\eta|(j, \ell) \mid<2.4$, and

(e) $|\Delta R(j j, \ell \ell, j \ell)|>0.4$,

where $p_{T}\left(\ell_{1}\right) \geq p_{T}\left(\ell_{2}\right)$ and $\Delta R \equiv \sqrt{(\Delta \phi)^{2}+(\Delta \eta)^{2}}$. It is clear to see that $M_{j_{1} j_{2}}$ distribution peaks at $m_{W}$, while $d \sigma / d M_{b j j}$ has a peak at $m_{t}$. 


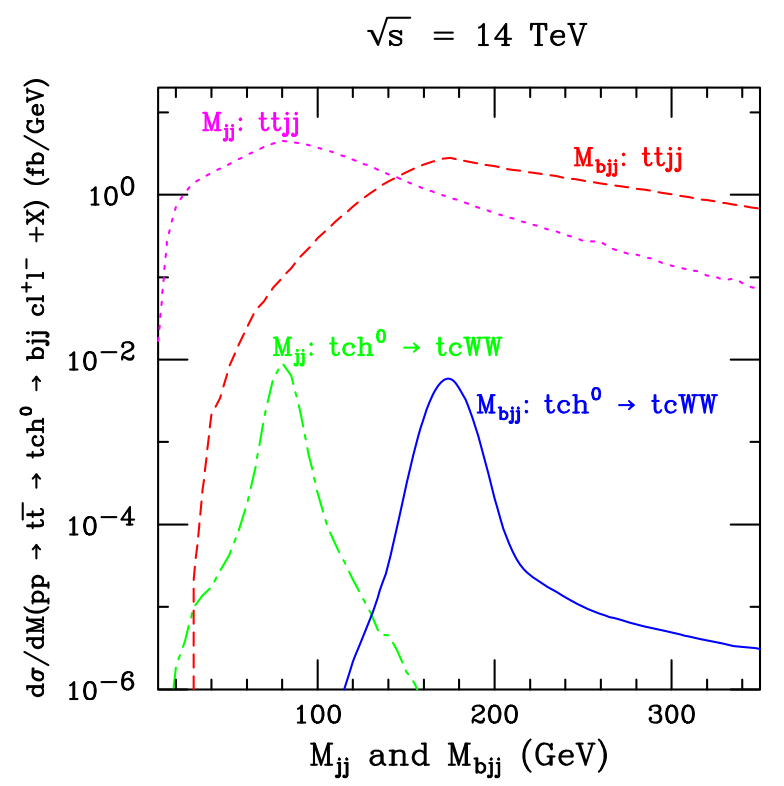

FIG. 2. Invariant mass distributions $(d \sigma / d M)$ of $j_{1} j_{2}$ (green dotted dashed) and $b j_{1} j_{2}$ (blue solid), for the Higgs signal in pp collisions, $\quad d \sigma / d M\left(p p \rightarrow t \bar{t} \rightarrow t c h^{0} \rightarrow t c W W \rightarrow\right.$ bjjcl $\ell^{+} \ell^{-}+$ $E_{T}+X(\mathrm{fb} / \mathrm{GeV})$, with basic cuts defined in Eq. (22). Also shown are the invariant mass distributions $d \sigma / d M_{j 1 j 2}$ (magenta dotted) and $d \sigma / d M_{b j 1 j 2}$ (red dashed) for the dominant physics background from $t \bar{t} j j$.

In a good reconstruction, the remaining light jet, $j_{3} \sim c$, should reproduce the top-quark mass with the momenta of charged leptons and neutrinos. To reconstruct the Higgs mass and top mass for $t \rightarrow c h^{0} \rightarrow c \ell^{+} \ell^{-}+E_{T}$, we use cluster transverse mass [49-51] $M_{T}\left(\ell \ell, E_{T}\right)$ and $M_{T}\left(c \ell \ell, E_{T}\right)$, defined as

$$
\begin{aligned}
M_{T}^{2}\left(\ell \ell, E_{T}\right)= & \left(\sqrt{p_{T}^{2}(\ell \ell)+M_{\ell \ell}^{2}}+{E_{T}}^{2}\right. \\
& -\left(\vec{p}_{T}(\ell \ell)+\vec{E}_{T}\right)^{2},
\end{aligned}
$$

and

$$
\begin{aligned}
M_{T}^{2}\left(c \ell \ell, E_{T}\right)= & \left(\sqrt{p_{T}^{2}(c \ell \ell)+M_{c \ell \ell}^{2}}+E_{T}\right)^{2} \\
& -\left(\vec{p}_{T}(c \ell \ell)+\vec{E}_{T}\right)^{2},
\end{aligned}
$$

where $p_{T}(\ell \ell)$ or $p_{T}(c \ell \ell)$ is the total transverse momentum of all the visible particles and $M_{\ell \ell}$ or $M_{c \ell \ell}$ is the invariant mass.

Figure 3 presents the cluster transverse mass distributions $\left[d \sigma / d M_{T}\left(\ell \ell, E_{T}\right)\right]$ and $\left[d \sigma / d M_{T}\left(c \ell \ell, E_{T}\right)\right]$ for the Higgs signal in pp collisions, $d \sigma / d M_{T}(p p \rightarrow t \bar{t} \rightarrow$ $t c h^{0} \rightarrow t c W W \rightarrow b j j c \ell^{+} \ell^{-}+E_{T}+X(\mathrm{fb} / \mathrm{GeV})$. We have applied basic cuts defined in Eq. (22) as well as invariant mass cuts $\left|M_{j j}-m_{W}\right| \leq 0.15 \times m_{W}$ and $\left|M_{b j j}-m_{t}\right| \leq 0.20 \times m_{t}$. The cluster transverse mass

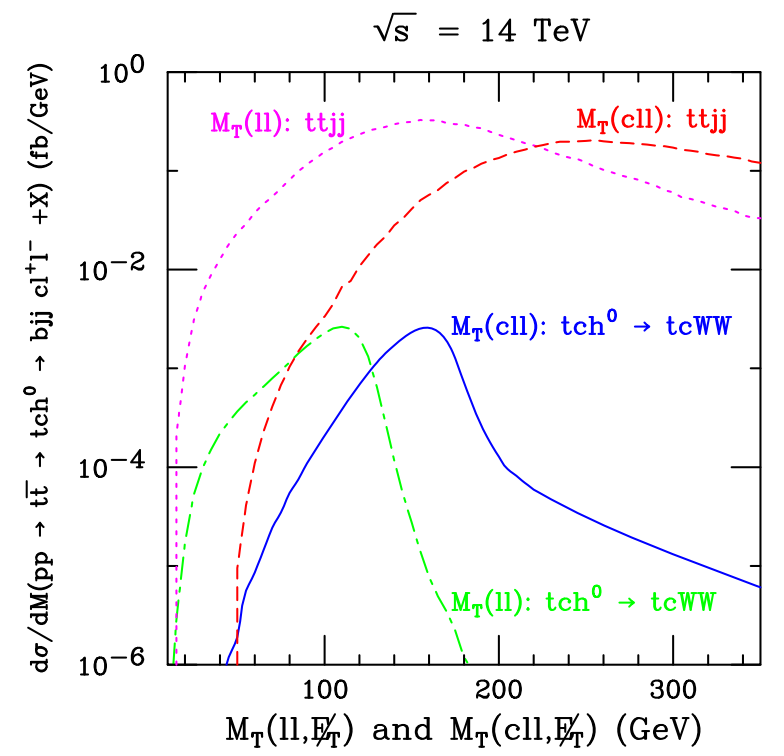

FIG. 3. Cluster transverse mass distributions $\left(d \sigma / d M_{T}\right)$ of $\ell^{+} \ell^{-}$(green dotted dashed) and $c \ell^{+} \ell^{-}$(blue solid) for the Higgs signal in pp collisions, $d \sigma / d M_{T}\left(p p \rightarrow t \bar{t} \rightarrow t c h^{0} \rightarrow\right.$ $t c W W \rightarrow$ bjjc $\ell^{+} \ell^{-}+E_{T}^{+}+X(\mathrm{fb} / \mathrm{GeV})$, with basic cuts defined in Eq. (22) as well as $\left|M_{j j}-m_{W}\right| \leq 0.15 \times m_{W}$ and $\left|M_{b j j}-m_{t}\right| \leq 0.20 \times m_{t}$. Also shown are the cluster transverse mass distributions $d \sigma / d M_{T}\left(\ell \ell, E_{T}\right)$ (magenta dotted) and $d \sigma / d M_{T}\left(c \ell \ell, E_{T}\right)$ (red dashed) for the dominant physics background from $t \bar{t} j j$.

distributions of $\ell^{+} \ell^{-}$and $c \ell^{+} \ell^{-}$are also shown for the dominant background $(t \bar{t} j j)$. Note that $d \sigma / d M_{T}\left(\ell \ell, E_{T}\right)$ peaks near $M_{h}$ while $d \sigma / d M_{T}\left(c \ell \ell, E_{T}\right)$ has a peak near $m_{t}$ for the signal of $t \rightarrow c h^{0}$.

It is clear that there are pronounced peaks at $m_{W}$ and $m_{t}$ in the invariant mass distributions of jets as shown in Fig. 2. We can also see broad peaks near $M_{h}$ and $m_{t}$ in the cluster transverse mass distributions,

$$
\begin{aligned}
M_{j_{1} j_{2}}^{*} & \simeq m_{W}, \quad M_{b j_{1} j_{2}}^{*} \simeq m_{t}, \\
M_{T}^{*}\left(\ell \ell, E_{T}\right) & \sim M_{h}, \quad M_{T}^{*}\left(c \ell \ell, E_{T}\right) \sim m_{t},
\end{aligned}
$$

where $M^{*}$ is the value of invariant mass or cluster transverse mass with a peak of the distribution. These distributions provide powerful selection tools to remove physics background while maintaining the Higgs signal.

\section{REALISTIC ACCEPTANCE CUTS}

To study the discovery potential of this charming FCNH signal from top decays at the LHC, we have applied realistic basic cuts listed in Eq. (22) and tagging efficiencies for $b$ jets. In addition to basic cuts, we apply cuts on the invariant mass of jets and cluster transverse mass of $\ell \ell$ and $c \ell \ell$ to effectively veto the background events:

(a) $\left|M_{j j}-m_{W}\right| \leq 0.15 \times m_{W}$,

(b) $\left|M_{b j j}-m_{t}\right| \leq 0.20 \times m_{t}$, 
TABLE II. Cross section of Higgs signal $p p \rightarrow t \bar{t} \rightarrow t c h^{0} \rightarrow$ bjjcle $+\mathscr{E}_{T}+X$ in femtobarns with $\cos (\beta-\alpha)=0.1$ for the LHC and future hadron colliders.

\begin{tabular}{lllll}
\hline \hline$\tilde{\rho}_{t c}$ & $13 \mathrm{TeV}$ & $14 \mathrm{TeV}$ & $27 \mathrm{TeV}$ & $100 \mathrm{TeV}$ \\
\hline 0.1 & 0.015 & 0.017 & 0.06 & 0.54 \\
0.5 & 0.364 & 0.425 & 1.53 & 13.6 \\
1 & 1.46 & 1.70 & 6.15 & 54.4 \\
\hline \hline
\end{tabular}

TABLE III. Cross section in femtobarns for dominant physics background processes, with $\mathrm{K}$ factors and tagging efficiencies at the LHC and future hadron colliders.

\begin{tabular}{lcccc}
\hline \hline Background & $13 \mathrm{TeV}$ & $14 \mathrm{TeV}$ & $27 \mathrm{TeV}$ & $100 \mathrm{TeV}$ \\
\hline $\mathrm{ttjj}$ & 14.6 & 17.1 & 63.6 & 557 \\
$\mathrm{ttW}$ & 0.16 & 0.17 & 0.36 & 1.41 \\
bbjj $\tau$ & 0.035 & 0.039 & 0.13 & 0.95 \\
bbjjWW & 0.003 & 0.0035 & 0.011 & 0.09 \\
ccjjWW & 0.0017 & 0.0019 & 0.006 & 0.05 \\
WWjjjj & $9.96 \mathrm{E}-06$ & $1.12 \mathrm{E}-05$ & $2.48 \mathrm{E}-05$ & 0.0002 \\
\hline \hline
\end{tabular}

(c) $50 \mathrm{GeV} \leq M_{T}\left(\ell \ell, E_{T}^{\dagger}\right) \leq 150 \mathrm{GeV}$,

(d) $100 \mathrm{GeV} \leq M_{T}\left(c \ell \ell, E_{T}\right) \leq 210 \mathrm{GeV}$.

These selection requirements remove more than $90 \%$ of the total background.

Measurement uncertainties in jet and lepton momenta as well as missing transverse momentum give rise to a spread in the reconstructed masses about the true values of $m_{t}$ and $M_{\phi}$. Based on the ATLAS [52] and CMS [53] specifications, we model these effects by Gaussian smearing of momenta,

(a) $\sqrt{\mathrm{s}}=13 \mathrm{TeV}, \cos (\beta-\alpha)=0.1$

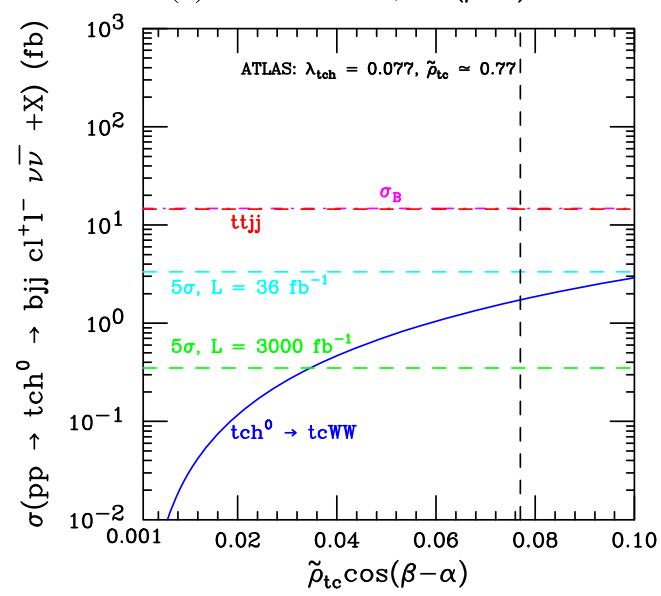

$$
\frac{\Delta E}{E}=\frac{0.60}{\sqrt{E(\mathrm{GeV})}} \oplus 0.03
$$

for jets and

$$
\frac{\Delta E}{E}=\frac{0.25}{\sqrt{E(\mathrm{GeV})}} \oplus 0.01
$$

for charged leptons with individual terms added in quadrature.

\section{DISCOVERY POTENTIAL AT THE LHC}

Applying all realistic cuts, we present our results for the Higgs signal at the LHC with $\sqrt{s}=13 \mathrm{TeV}$ and $\sqrt{s}=$ $14 \mathrm{TeV}$ as well as cross sections for future hadron colliders with $\sqrt{s}=27 \mathrm{TeV}$ and $\sqrt{s}=100 \mathrm{TeV}$ in Table II. Here, we have kept $\cos (\beta-\alpha)=0.1$. Later, we will vary it from 0.01 to 0.2 for discovery contours. Cross sections for dominant background processes are presented in Table III.

To estimate the discovery potential at the LHC, we include curves that correspond to the minimal cross section of signal $\left(\sigma_{S}\right)$ required by our discovery criterion described in the following. We define the signal to be observable if the lower limit on the signal plus background is larger than the corresponding upper limit on the background with statistical fluctuations

$L\left(\sigma_{S}+\sigma_{B}\right)-N \sqrt{L\left(\sigma_{S}+\sigma_{B}\right)} \geq L \sigma_{B}+N \sqrt{L \sigma_{B}}$,

or equivalently

(b) $\sqrt{\mathrm{s}}=14 \mathrm{TeV}, \cos (\beta-\alpha)=0.1$

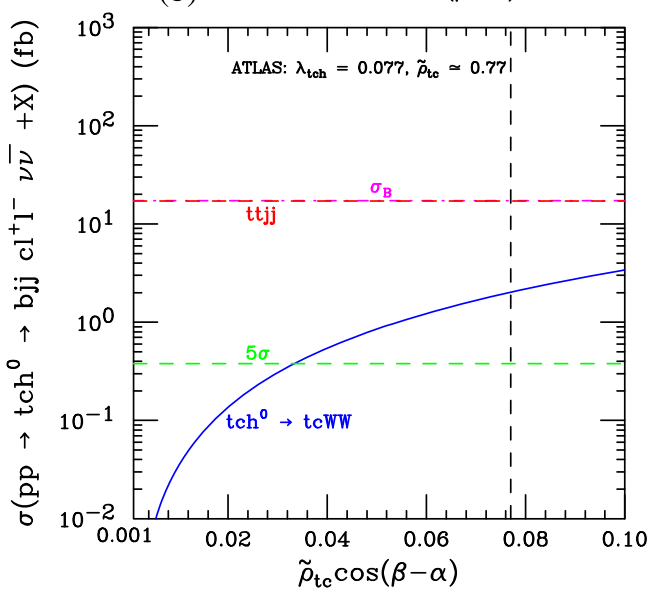

FIG. 4. The cross section in fb of $p p \rightarrow t \bar{t} \rightarrow t c h^{0} \rightarrow$ bjjc $\ell^{+} \ell^{-}+E_{T}+X$ at (a) $\sqrt{s}=13 \mathrm{TeV}$ and (b) $\sqrt{s}=14 \mathrm{TeV}$ as a function of $\tilde{\rho}_{t c}$, along with the total (magenta dotted dashed) and most dominant (red dashed) background after applying all the cuts, tagging, and mistagging efficiencies and higher-order QCD corrections. The blue dashed line and green dashed line show the minimum cross section needed for $5 \sigma$ significance at $L=36 \mathrm{fb}^{-1}$ and $3 \mathrm{ab}^{-1}$, respectively, for a c.m. energy of $13 \mathrm{TeV}$, whereas for $14 \mathrm{TeV}$, we present $L=3 \mathrm{ab}^{-1}$ (green dashed) only. The current ATLAS limit [34] is shown as a black dashed vertical line. 


$$
\sigma_{S} \geq \frac{N}{L}\left[N+2 \sqrt{L \sigma_{B}}\right]
$$

Here, $L$ is the integrated luminosity, $\sigma_{S}$ is the cross section of the FCNH signal, and $\sigma_{B}$ is the background cross section. The parameter $N$ specifies the level or probability of discovery. We take $N=2.5$, which corresponds to a $5 \sigma$ signal.

For $L \sigma_{B} \gg 1$, this requirement becomes similar to

$$
N_{\mathrm{SS}}=\frac{N_{S}}{\sqrt{N_{B}}}=\frac{L \sigma_{S}}{\sqrt{L \sigma_{B}}} \geq 5,
$$

where $N_{S}$ is the signal number of events, $N_{B}$ is the background number of events, and $N_{\mathrm{SS}}$ is the statistical significance, which is commonly used in the literature. If the background has fewer than 25 events for a given luminosity, we employ the Poisson distribution and require that the Poisson probability for the SM background to fluctuate to this level is less than $2.87 \times 10^{-7}$, i.e., an equivalent probability to a $5 \sigma$ fluctuation with Gaussian statistics.

Figure 4 shows the Higgs signal cross section as a function of $\tilde{\rho}_{t c}$, along with cross section of total background and the most dominant background process $(t t j j)$ for the CERN Large Hadron Collider with (a) $\sqrt{s}=13 \mathrm{TeV}$ and (b) $\sqrt{s}=14 \mathrm{TeV}$. We have also shown the minimum cross section required for $5 \sigma$ significance at $L=36.1 \mathrm{fb}^{-1}$ and higher luminosities for the future HL LHC [36,37], i.e., $L=300$ and $3000 \mathrm{fb}^{-1}$. All tagging efficiencies and $\mathrm{K}$
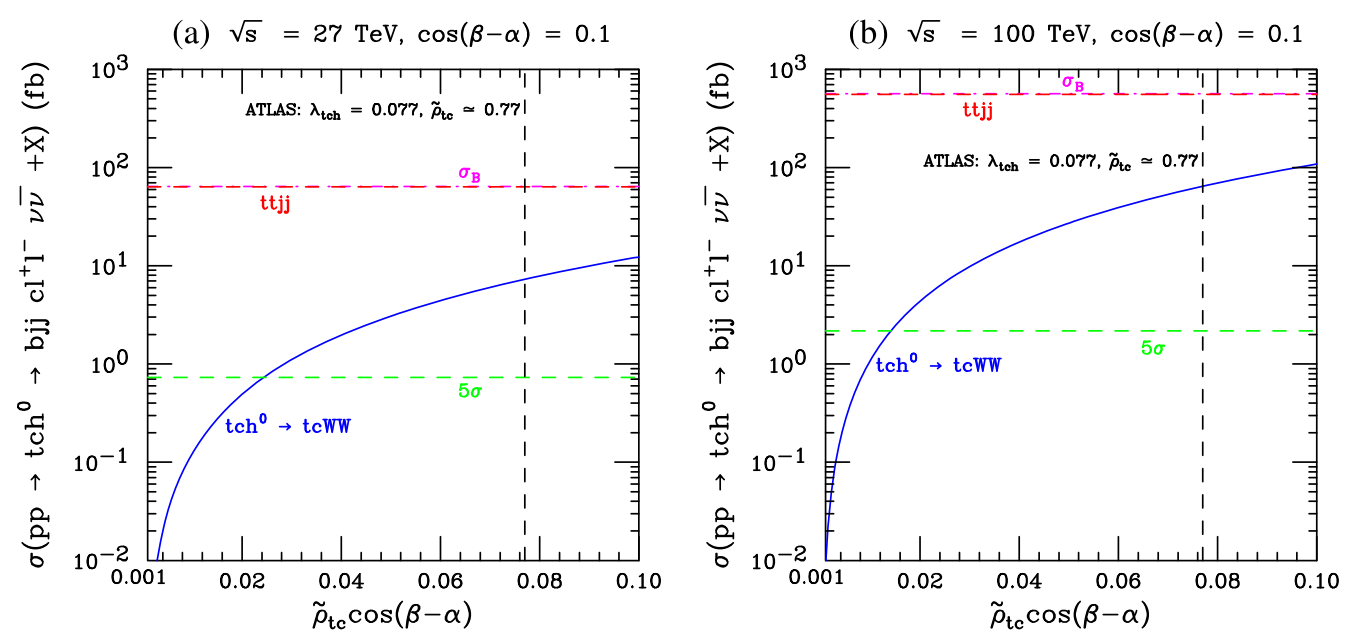

FIG. 5. Similar to Fig. 4, but for (a) $\sqrt{s}=27 \mathrm{TeV}$ and (b) $100 \mathrm{TeV}$.

(a) $\sqrt{s}=13 \mathrm{TeV}$

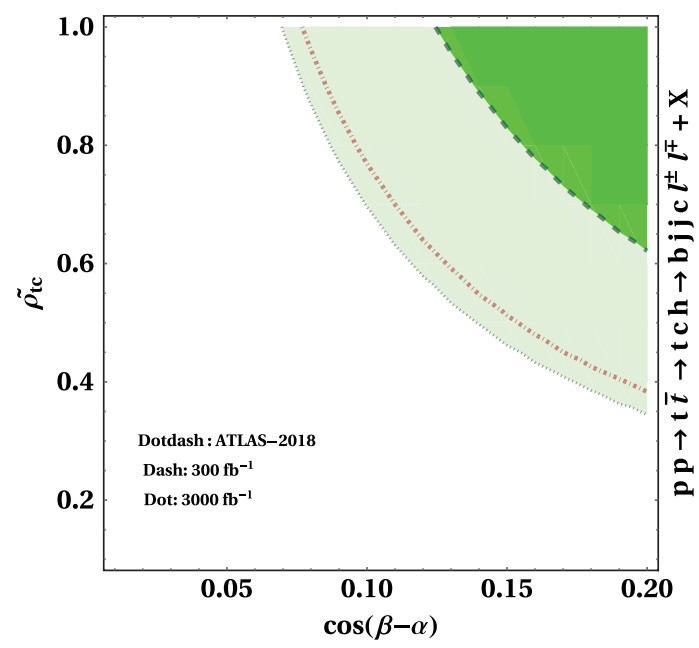

(b) $\sqrt{s}=14 \mathrm{TeV}$

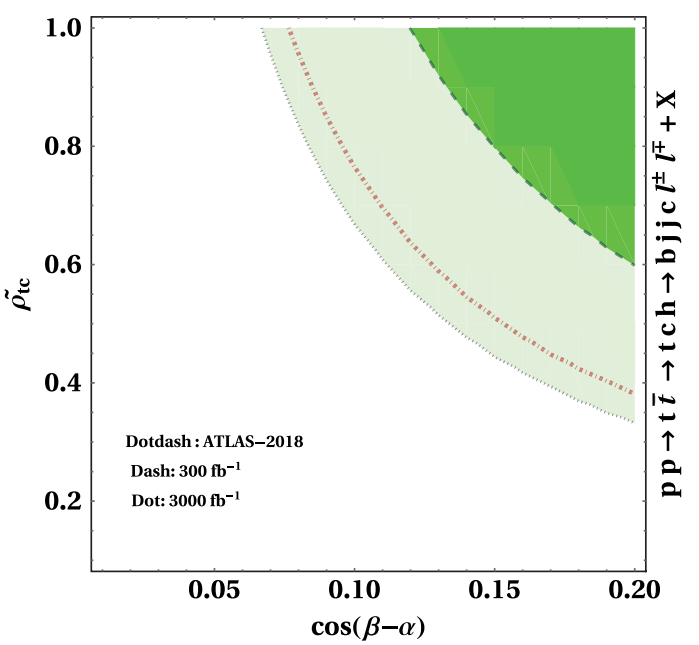

FIG. 6. The $5 \sigma$ discovery contours at the LHC in the plane of $\left[\cos (\beta-\alpha), \tilde{\rho}_{t c}\right]$ for (a) $\sqrt{s}=13 \mathrm{TeV}$ and (b) $\sqrt{s}=14 \mathrm{TeV}$, with $L=300 \mathrm{fb}^{-1}$ (dashed) and $L=3000 \mathrm{fb}^{-1}$ (dotted). Also shown is the current limit on $\lambda_{t c h}=\tilde{\rho}_{t c} \cos (\beta-\alpha)$ (red dotted dashed) set by ATLAS [34]. The shaded region above this curve is excluded at $95 \%$ C.L. 
(a) $\sqrt{s}=27 \mathrm{TeV}$

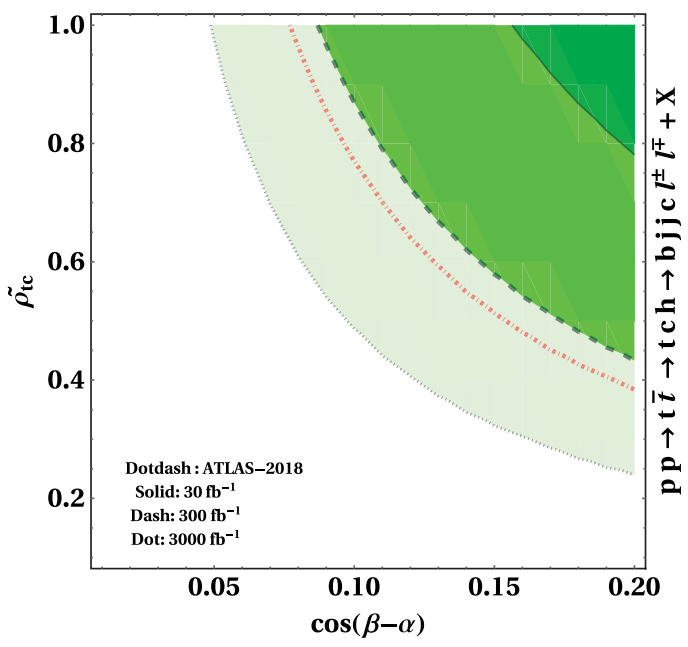

(b) $\sqrt{s}=100 \mathrm{TeV}$

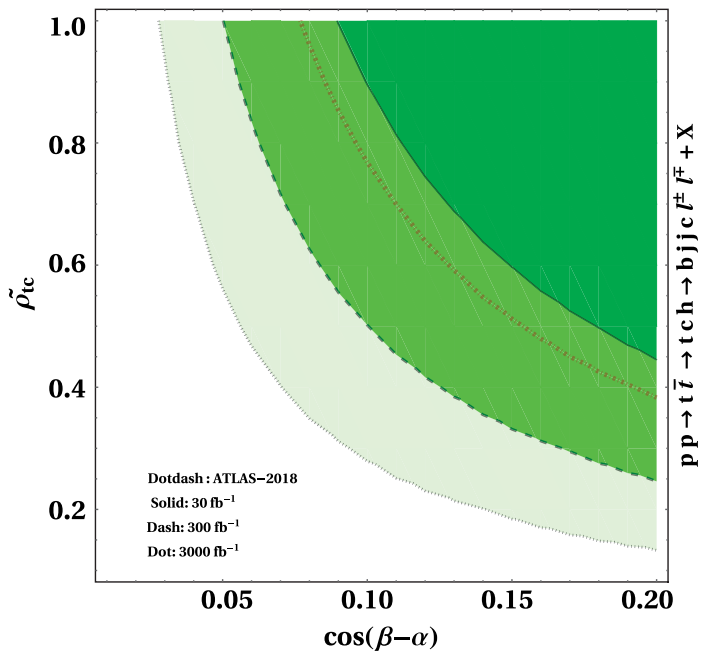

FIG. 7. The $5 \sigma$ discovery contours at future pp colliders in the plane of $\left[\cos (\beta-\alpha), \tilde{\rho}_{t c}\right]$ for (a) $\sqrt{s}=27 \mathrm{TeV}$ and (b) $\sqrt{s}=100 \mathrm{TeV}$, for $L=30 \mathrm{fb}^{-1}$ (solid), $L=300 \mathrm{fb}^{-1}$ (dashed), and $L=3000 \mathrm{fb}^{-1}$ (dotted). Also shown is the current limit on $\lambda_{t c h}=\tilde{\rho}_{t c} \cos (\beta-\alpha)$ (red dotted dashed) set by ATLAS [34]. The shaded region above this curve is excluded at 95\% C.L.

factors discussed above are included. Our analysis suggests an improvement in the reach of ATLAS at a luminosity of $3000 \mathrm{fb}^{-1}$, which gets better at higher energies, i.e., $\sqrt{s}=$ 27 and $100 \mathrm{TeV}$, as shown in Fig. 5.

We present the $5 \sigma$ discovery reach at the LHC for (a) $\sqrt{s}=13 \mathrm{TeV}$ and (b) $\sqrt{s}=14 \mathrm{TeV}$ in Fig. 6, in the parameter plane of $\left[\cos (\beta-\alpha), \tilde{\rho}_{t c}\right]$. We have chosen $L=300$ and $3000 \mathrm{fb}^{-1}$. Figure 7 shows the discovery contours for (a) $\sqrt{s}=27 \mathrm{TeV}$ and (b) $\sqrt{s}=100 \mathrm{TeV}$. The high-energy (HE) LHC with high luminosity is quite promising as it nearly covers the entire parameter space that we have used in our analysis.

\section{CONCLUSIONS}

It is a generic possibility of particle theories beyond the Standard Model to have contributions to tree-level FCNH interactions, especially for the third-generation quarks. These contributions arise naturally in models with additional Higgs doublets, such as the special two Higgs doublet model for the top quark [10] or a general 2HDM. In the alignment limit, the light Higgs boson $\left(h^{0}\right)$ resembles the standard Higgs boson, and it has a mass below the top mass. This could engender the rare decay $t \rightarrow c h^{0}$.

We investigated the prospects for discovering such a decay at the LHC, focusing on the channel in which $t \bar{t}$ are pair produced and subsequently decay, one hadronically and the other through the FCNH mode. The primary background for this signal is a $t \bar{t} j j$ with both top quarks decaying leptonically. This background involves one $b$ jet mistagged as a $c$ jet and two other light jets, along with two leptons and missing transverse energy. Nonetheless, by taking advantage of the available kinematic information, we can reconstruct the resonances of the signal and reject much of the background.

Based on our analysis, we find that the LHC at $\sqrt{s}=14 \mathrm{TeV}$, with $L=3000 \mathrm{fb}^{-1}$, can probe as low as $\mathcal{B}\left(t \rightarrow c h^{0}\right) \simeq 1.17 \times 10^{-3}, \lambda_{t c h}=\tilde{\rho}_{t c} \cos (\beta-\alpha) \simeq 0.069$. It gets better with $\sqrt{s}=27 \mathrm{TeV}$ and $\sqrt{s}=100 \mathrm{TeV}$, which can reach up to $\mathcal{B}\left(t \rightarrow c h^{0}\right) \simeq 6.1 \times 10^{-4}, \lambda_{\text {tch }} \simeq 0.048$ and $\mathcal{B}\left(t \rightarrow c h^{0}\right) \simeq 2 \times 10^{-4}, \lambda_{t c h} \simeq 0.028$, respectively.

We look forward to being guided by more new experimental results as we explore interesting physics of electroweak symmetry breaking and FCNH interactions. While the properties of the Higgs boson go under further scrutiny as data accumulate, perhaps a dedicated FCNH $t \rightarrow c h^{0}$ search should be undertaken, for upcoming HL LHC and further HE LHC as well as future high-energy hadron colliders with a c.m. energy of $100 \mathrm{TeV}$.

\section{ACKNOWLEDGMENTS}

We are grateful to Kai-Feng Jack Chen for beneficial discussions. C. K. thanks the Institute of Physics at the Academia Sinica and the High Energy Physics Group at National Taiwan University, where part of the research was completed, for excellent hospitality. This research was supported in part by the U.S. Department of Energy under Grant No. DE-FG02-13ER41979. 
[1] G. Aad et al. (ATLAS Collaboration), Phys. Lett. B 716, 1 (2012).

[2] S. Chatrchyan et al. (CMS Collaboration), Phys. Lett. B 716, 30 (2012).

[3] J. A. Aguilar-Saavedra, Acta Phys. Pol. B 35, 2695 (2004).

[4] B. Mele, S. Petrarca, and A. Soddu, Phys. Lett. B 435, 401 (1998).

[5] G. Eilam, J. L. Hewett, and A. Soni, Phys. Rev. D 44, 1473 (1991); 59, 039901(E) (1998).

[6] S. L. Glashow and S. Weinberg, Phys. Rev. D 15, 1958 (1977).

[7] J. F. Gunion, H. E. Haber, G. L. Kane, and S. Dawson, Front. Phys. 80, 1 (2000).

[8] W. S. Hou, Phys. Lett. B 296, 179 (1992).

[9] T. P. Cheng and M. Sher, Phys. Rev. D 35, 3484 (1987).

[10] A. K. Das and C. Kao, Phys. Lett. B 372, 106 (1996).

[11] S. Davidson and H. E. Haber, Phys. Rev. D 72, 035004 (2005); 72, 099902 (2005).

[12] F. Mahmoudi and O. Stal, Phys. Rev. D 81, 035016 (2010).

[13] P. Nason, S. Dawson, and R. K. Ellis, Nucl. Phys. B303, 607 (1988).

[14] N. Kidonakis, Phys. Rev. D 82, 114030 (2010).

[15] V. Ahrens, A. Ferroglia, M. Neubert, B. D. Pecjak, and L. L. Yang, Phys. Lett. B 703, 135 (2011).

[16] M. Cacciari, M. Czakon, M. Mangano, A. Mitov, and P. Nason, Phys. Lett. B 710, 612 (2012).

[17] M. Czakon, P. Fiedler, and A. Mitov, Phys. Rev. Lett. 110, 252004 (2013).

[18] M. Aaboud et al. (ATLAS Collaboration), Phys. Lett. B 761, 136 (2016); 772, 879(E) (2017).

[19] A. M. Sirunyan et al. (CMS Collaboration), J. High Energy Phys. 09 (2017) 051.

[20] S. Heinemeyer et al. (LHC Higgs Cross Section Working Group), arXiv:1307.1347.

[21] G. Aad et al. (ATLAS Collaboration), Eur. Phys. J. C 76, 6 (2016).

[22] ATLAS and CMS Collaborations, Report No. ATLASCONF-2015-044.

[23] J. F. Gunion and H.E. Haber, Phys. Rev. D 67, 075019 (2003).

[24] M. Carena, I. Low, N. R. Shah, and C. E. M. Wagner, J. High Energy Phys. 04 (2014) 015.

[25] C. Kao, H. Y. Cheng, W. S. Hou, and J. Sayre, Phys. Lett. B 716, 225 (2012).

[26] D. Atwood, S. K. Gupta, and A. Soni, J. High Energy Phys. 10 (2014) 057.

[27] A. M. Sirunyan et al. (CMS Collaboration), J. High Energy Phys. 06 (2018) 102.
[28] S. Banerjee, M. Chala, and M. Spannowsky, Eur. Phys. J. C 78, 683 (2018).

[29] K. F. Chen, W. S. Hou, C. Kao, and M. Kohda, Phys. Lett. B 725, 378 (2013).

[30] M. Aaboud et al. (ATLAS Collaboration), J. High Energy Phys. 10 (2017) 129.

[31] N. Craig, J. A. Evans, R. Gray, M. Park, S. Somalwar, S. Thomas, and M. Walker, Phys. Rev. D 86, 075002 (2012).

[32] CMS Collaboration, Report No. CMS-PAS-HIG-13-034.

[33] V. Khachatryan et al. (CMS Collaboration), Phys. Rev. D 90, 112013 (2014).

[34] M. Aaboud et al. (ATLAS Collaboration), Phys. Rev. D 98, 032002 (2018).

[35] ATLAS Collaboration, Report No. ATLAS-PHYS-PUB2013-012.

[36] W. Barletta, M. Battaglia, M. Klute, M. Mangano, S. Prestemon, L. Rossi, and P. Skands, arXiv:1310.0290.

[37] R. Tomas et al., Nuclear and Particle Physics Proceedings 273, 149 (2016).

[38] F. Zimmermann, ICFA Beam Dyn. Newslett. 72, 138 (2017).

[39] V. Shiltsev, arXiv:1705.02011.

[40] B. Altunkaynak, W. S. Hou, C. Kao, M. Kohda, and B. McCoy, Phys. Lett. B 751, 135 (2015).

[41] J. Alwall, M. Herquet, F. Maltoni, O. Mattelaer, and T. Stelzer, J. High Energy Phys. 06 (2011) 128.

[42] K. Hagiwara, J. Kanzaki, Q. Li, and K. Mawatari, Eur. Phys. J. C 56, 435 (2008).

[43] K. Hagiwara, J. Kanzaki, H. Murayama, and I. Watanabe, Eur. Phys. J. C 56, 435 (2008).

[44] S. Dulat, T.-J. Hou, J. Gao, M. Guzzi, J. Huston, P. Nadolsky, J. Pumplin, C. Schmidt, D. Stump, and C.-P. Yuan, Phys. Rev. D 93, 033006 (2016).

[45] J. Gao, M. Guzzi, J. Huston, H.-L. Lai, Z. Li, P. Nadolsky, J. Pumplin, D. Stump, and C.-P. Yuan, Phys. Rev. D 89, 033009 (2014).

[46] ATLAS Collaboration, Report No. ATLAS-CONF-2018001.

[47] L. Scodellaro (ATLAS and CMS Collaborations), arXiv: 1709.01290.

[48] CMS Collaboration, Report No. CMS-PAS-HIG-15-008.

[49] V. Barger and R. J. N.Phillips, Frontiers in Physics Vol. 71 (Addison-Wesley, Redwood City, USA, 1987), p. 592, ISBN: 0-201-14945-1.

[50] T. Han and R. J. Zhang, Phys. Rev. Lett. 82, 25 (1999).

[51] C. Kao and J. Sayre, Phys. Lett. B 722, 324 (2013).

[52] ATLAS Collaboration, Report No. ATLAS-PHYS-PUB2013-004.

[53] CMS Collaboration, J. Instrum. 12, P02014 (2017). 